\title{
Review of Transmetatarsal Amputations in the Management of Peripheral Arterial Disease in an Asian Population
}

\author{
Ming Ngan Aloysius Tan, MBBS, MRCS, ${ }^{1}$ Zhiwen Joseph Lo, MBBS, MRCS, FRCS, ${ }^{1}$ Soon Hong \\ Lee, MBBS, ${ }^{2}$ Rui Ming Teo, MBBS, MRCS, ${ }^{1}$ Wei Leong Glenn Tan, MBBS, MRCS, FRCS, ${ }^{1}$ and \\ Sadhana Chandrasekar, MBBS, MRCS, FRCS1
}

Objective: To evaluate outcomes after transmetatarsal amputation (TMA) in peripheral arterial disease (PAD) limb salvage in an Asian population and identify risk factors associated with TMA failure.

Methodology: A retrospective review of 147 patients with PAD, who had undergone TMA between 2008 and 2014, was carried out. Univariate and multivariate analysis were used to identify predictors of TMA failure. Kaplan-Meier survival analysis was used to calculate major amputation and all-cause mortality rates.

Results: The mean age was 66 years. 92\% were diabetic patients and $78 \%$ had preceded angioplasty. $56 \%$ of TMAs were healed via secondary intention, $8 \%$ required subsequent split-thickness skin graft closure, $24 \%$ required further debridement while $37 \%$ had wounds, which failed to heal and required below-knee amputations (BKA). Multivariate analysis showed that diabetes is the only independent predictor of TMA failure (odds ratio $(O R)$ 7.11, $\mathrm{p}=0.064$ ). Patients with TMA failure were at increased risk of developing nosocomial infections $(p=0.025)$ and faced a higher risk of 30-day re-admission rate $(p=0.002)$.

Conclusion: The success rate for PAD limb salvage TMA was $63 \%$ and diabetes was an independent predictor of TMA failure. Patients with TMA failure were at increased risks of nosocomial infections, and 30-day re-admissions;

${ }^{1}$ Vascular Surgery Service, Department of General Surgery, Tan Tock Seng Hospital, Singapore

${ }^{2}$ Lee Kong Chian School of Medicine, National Technological University, Singapore

Received: November 22, 2017; Accepted: February 15, 2018 Corresponding author: Ming Ngan Aloysius Tan, MBBS, MRCS. Vascular Surgery Service, Department of General Surgery, Tan Tock Seng Hospital, 11 Jalan Tan Tock Seng, Singapore 308433 Tel: +65-9017-7634, Fax: +65-6252-7282

E-mail: tanaloysius@gmail.com

(cc) BY-NC-SA (02018 The Editorial Committee of Annals of Vascular Diseases. This article is distributed under the terms of the Creative Commons Attribution License, which permits use, distribution, and reproduction in any medium, provided the credit of the original work, a link to the license, and indication of any change are properly given, and the original work is not used for commercial purposes. Remixed or transformed contributions must be distributed under the same license as the original. hence the risks and benefits of TMA for diabetic foot limb salvage must be individualized for each patient.

Keywords: peripheral arterial disease, limb salvage, diabetic foot, amputation, transmetatarsal amputation

\section{Introduction}

Peripheral arterial disease (PAD) affects more than 200 million people worldwide and it is associated with significant rates of cardiovascular morbidity and mortality. It is the third leading cause of atherosclerotic vascular morbidity after coronary heart disease and stroke. ${ }^{1,2}$ ) This disease burden is expected to increase further, with increased exposure to chronic risk factors and an aging population. ${ }^{3)}$ Patients with PAD also face about a threefold higher risk of mortality and major cardiovascular events. ${ }^{4)}$

PAD affects about 1 in 10 people aged 70 and 1 in 6 people older than 80 years old.5) The true incidence of the disease may be higher than the estimated number given that most patients are asymptomatic and have a screening ankle-brachial index (ABI) of 0.90 with a sensitivity of less than $80 \% .^{6}$ In Asia, the higher incidence of diabetes mellitus (DM) has aggravated a greater incidence of peripheral vascular disease. ${ }^{7)}$ The combination of PAD and $\mathrm{DM}$ is associated with significantly poorer healing rates and risks of limb loss. ${ }^{8)}$

In patients with PAD and forefoot sepsis or tissue loss, transmetatarsal amputation (TMA) is a viable option to avoid major amputation of a lower limb. TMA, compared to a below-knee amputation (BKA), does not require a prosthesis and has a lower energy expenditure. ${ }^{9)}$ However, TMAs are associated with significant failure or reamputation rates of between $26 \%$ and $63 \% .{ }^{9,10)}$ Although previous studies have attempted to identify the risk factors associated with failure of TMA, some are found to be conflicting. Many of the studies evaluating TMA were conducted in western populations. Hence, this study aims to evaluate the outcomes of TMA in the Asian population 
and identify risk factors associated with TMA failure.

\section{Methods}

\section{Study cohort}

A retrospective study was conducted in 147 patients with PAD who underwent TMA between 2008 and 2014 at a 1,500-bed university tertiary hospital. Patients whose surgery was performed for other reasons, such as trauma or malignancies, were excluded.

\section{Management of PAD}

A multi-disciplinary team headed by the vascular surgeons manages patients with PAD. Pre-operatively, vasculature was evaluated by ABI with toe pressures, either with arterial duplex imaging or computed tomography $(\mathrm{CT})$ angiogram. Revascularisation modalities include both endovascular and open bypasses. For patients with foot sepsis or tissue loss, empirical antibiotics were carried out in accordance with the hospital's antibiogram and subsequently switched to targeted therapy according to cultures.

The decision for a TMA was made only if the patient did not have tissue loss proximal to the midfoot, had adequate vascularity to the infra-popliteal region and assessed to have ambulatory potential after amputation. Patients not fulfilling these criteria were offered a more proximal amputation, either below or above the level of the knee. We do not perform ankle disarticulation amputations in our institution for patients with PAD. Revascularisation was performed with either open (open bypass) or endovascular methods (percutaneous angioplasty). Revascularisation was offered to patients with toe pressures of $<50 \mathrm{mmHg}$ with the issue of tissue loss, or in selected cases with poor wound healing and severe disease shown on preoperative imaging. A consultant Interventional radiologist or vascular surgeon performed angioplasty. Moreover, TMAs were performed in a guillotine fashion, ensuring a complete debridement of non-viable tissue before leaving the wounds to heal via secondary intention. None of our study population had their TMA wounds closed primarily. Negative pressure wound therapy (NPWT) is applied to these wounds immediately patients are discharged. Home NPWT was also carried out for at least 2 to 6 weeks before an outpatient evaluation in the clinic. Split skin grafts are performed for granulating wounds to expedite healing. All amputations were performed by vascular surgeons.

\section{Outcomes}

The primary outcome of the study was the failure of an initial TMA, defined as patients required a subsequent BKA (trans-tibial amputation) because of the failure of wound healing. Secondary outcomes evaluated include a need for subsequent debridement or split skin grafting, post-operative complications (acute myocardial infarction, cerebral vascular accident, acute kidney injury, and nosocomial infections), 30-day re-admission rates and allcause mortality. Multivariate analysis was used to identify the risk factors predicting the TMA failure.

In accordance with the Singapore Ministry of Health's guidelines, hypertension was defined as a blood pressure level $\geq 140 / 90$, having diabetes at a 2-hour oral glucose tolerance test $\geq 11.1 \mathrm{mmol} / \mathrm{L}$, and hyperlipidemia at a total cholesterol $\geq 6.2 \mathrm{mmol} / \mathrm{L}$. End-stage renal failure was defined as patients were offered renal replacement therapy. Ischemic heart disease was recorded for patients who had a previous myocardial infarction or documented cardiac investigation revealing significant cardiac disease. Visual impairment was recorded for patients who had documented diabetic retinopathy in a follow-up.

\section{Statistical Analysis}

Descriptive statistics were used to evaluate demographics and provide the results of the present investigation. Percentages were used for categorical data and means with standard deviations (SD) for continuous data. Comparisons between groups for categorical data were carried out with the aid of chi-square tests while continuous data were done using Student's t-test. Univariate analysis was performed to identify factors associated with TMA's failure, using a cutoff p-value of 0.2. Moreover, we used multivariate logistic regression to identify independent risk factors associated with TMA failure. Odds ratios (OR) and their $95 \%$ confidence intervals (CI) were calculated. All p-values $<0.05$ were considered statistically significant and p-values were two-tailed. SPSS 17.0 (Chicago, IL, USA) was used for the statistical analysis.

\section{Results}

A total of 147 patients underwent TMA during the study period (Table 1). The mean age was 65.9 (range 43-85). The majority were male $(69 \%)$; and the racial distribution matched the local population with the majority being Chinese $(69 \%)$. The majority of patients had significant co-morbidities, with 122 patients ( $85 \%$ ) having an American Society of Anesthesiologist (ASA) status classification of 3 or 4 . Although only $26 \%$ were smokers, the study population had a larger proportion of co-morbidities than the local population because of a higher incidence of smoking. ${ }^{11)}$ Type II DM and hypertension were present in $92 \%$ and $94 \%$ of patients, respectively, compared to only $11 \%$ and $24 \%$ in the general population. More than half $(53 \%)$ of the patients with DM had the sub-optimal disease of a HbA1c level of $>7 \%$. More than two-thirds 
Table 1 Univariate and multivariate analysis of factors predicting failure of TMA

\begin{tabular}{|c|c|c|c|c|}
\hline TMA $(n=147)$ & $\begin{array}{l}\text { TMA failure } \\
(n=54,37 \%)\end{array}$ & $\begin{array}{l}\text { TMA success } \\
(n=93,63 \%)\end{array}$ & $\begin{array}{l}\text { Univariate } \\
p \text {-value }\end{array}$ & Multivariate \\
\hline \multicolumn{5}{|l|}{ Demographics } \\
\hline Mean age (range) & $65.1(43-84)$ & $66.4(49-85)$ & 0.467 & \\
\hline Male & $34(63 \%)$ & $67(72 \%)$ & 0.252 & \\
\hline Ethnicity & & & 0.723 & \\
\hline - Chinese & $39(72 \%)$ & $63(68 \%)$ & & \\
\hline - Malay & $9(17 \%)$ & $17(18 \%)$ & & \\
\hline - Indian & $6(11 \%)$ & $11(12 \%)$ & & \\
\hline - Others & 0 & $2(2 \%)$ & & \\
\hline \multicolumn{5}{|l|}{ Co-morbidities } \\
\hline Smoker & $15(28 \%)$ & $23(25 \%)$ & 0.244 & \\
\hline Diabetes & $53(98 \%)$ & $82(88 \%)$ & $\underline{0.033}$ & $\underline{0.032}$ \\
\hline $\mathrm{HbA} 1 \mathrm{c}>7 \%$ & $31(57 \%)$ & $47(51 \%)$ & 0.576 & \\
\hline Hypertension & $52(96 \%)$ & $86(93 \%)$ & 0.351 & \\
\hline Hyperlipidemia & $49(91 \%)$ & $77(83 \%)$ & 0.184 & 0.236 \\
\hline Ischemic heart disease & $23(43 \%)$ & $51(55 \%)$ & 0.152 & 0.131 \\
\hline Ejection fraction $<55 \%$ & $14(26 \%)$ & $27 / 93(29 \%)$ & 0.244 & \\
\hline ESRF & $15(28 \%)$ & $22(24 \%)$ & 0.579 & \\
\hline Visual impairment & $18(33 \%)$ & $33(36 \%)$ & 0.792 & \\
\hline Previous amputation & $37(69 \%)$ & $64(69 \%)$ & 0.970 & \\
\hline ASA & & & 0.633 & \\
\hline-2 & $9(17 \%)$ & $13(14 \%)$ & & \\
\hline-3 & $41(76 \%)$ & $76(82 \%)$ & & \\
\hline-4 & $4(7 \%)$ & $4(4 \%)$ & & \\
\hline \multicolumn{5}{|l|}{ Clinical presentation } \\
\hline Ulcer & $13(24 \%)$ & $20(22 \%)$ & 0.719 & \\
\hline Wet gangrene & $35(65 \%)$ & $79(85 \%)$ & 0.228 & \\
\hline Dry gangrene & $17(32 \%)$ & $22(24 \%)$ & 0.300 & \\
\hline \multicolumn{5}{|l|}{ Wound culture } \\
\hline Positive vs. negative & $41(76 \%)$ & $60(65 \%)$ & 0.150 & 0.343 \\
\hline$>1$ organism & $19(35 \%)$ & $23(25 \%)$ & 0.255 & \\
\hline MRSA & $7(13 \%)$ & $7(8 \%)$ & 0.261 & \\
\hline Pseudomonas & $11(20 \%)$ & $12(13 \%)$ & 0.254 & \\
\hline Toe Pressure $<50 \mathrm{mmHg}$ & $42(78 \%)$ & $75(81 \%)$ & 0.173 & \\
\hline \multicolumn{5}{|c|}{ Below-knee patency post-revascularisation } \\
\hline ATA & $22(41 \%)$ & $43(46 \%)$ & 0.366 & \\
\hline PTA & $18(33 \%)$ & $40(43 \%)$ & 0.160 & 0.106 \\
\hline Peroneal & $29(54 \%)$ & $49(53 \%)$ & 0.883 & \\
\hline Plantar arch & $17(32 \%)$ & $27(29 \%)$ & 0.874 & \\
\hline \multicolumn{5}{|c|}{ Below-knee patency post-revascularisation } \\
\hline 3-vessels & $7(13 \%)$ & $16(17 \%)$ & 0.485 & \\
\hline 2-vessels & $15(28 \%)$ & $27(29 \%)$ & 0.936 & \\
\hline 1-vessel & $22(40.7 \%)$ & $31(33.3 \%)$ & 0.773 & \\
\hline
\end{tabular}

of these patients $(69 \%)$ had a previous minor or a major lower limb amputation.

A large number of patients developed wet gangrene $(71 \%)$. Wound cultures were positive in $69 \%$ of the patients, with $29 \%$ having multiple bacteria isolated. Preoperative toe pressures were measured, with 117 patients $(79.6 \%)$ having toe pressures of $<50 \mathrm{mmHg}$. Only two
Table 2 Outcomes of TMA

\begin{tabular}{lc}
\hline \multicolumn{1}{c}{ Outcome } & Results $(\mathrm{n}=147)$ \\
\hline Further amputation & $54(36.7 \%)$ \\
Debridement & $35(23.8 \%)$ \\
Split skin graft & $11(7.5 \%)$ \\
\hline
\end{tabular}


Table 3 Complications after TMA

\begin{tabular}{lccc}
\hline \multicolumn{1}{c}{ TMA $(\mathrm{n}=147)$} & TMA failure $(\mathrm{n}=54,37 \%)$ & TMA success $(\mathrm{n}=93,63 \%)$ & Univariate $\mathrm{p}$-value \\
\hline Acute myocardial infarction & $10(19 \%)$ & $8(9 \%)$ & 0.077 \\
Cerebrovascular accident & $3(6 \%)$ & $5(5 \%)$ & 0.963 \\
Acute kidney injury & $7(13 \%)$ & $6(7 \%)$ & 0.180 \\
Nosocomial infection & $15(28 \%)$ & $12(13 \%)$ & $\underline{\mathbf{0 . 0 2 5}}$ \\
30-Day re-admission & $17(32 \%)$ & $10(11 \%)$ & $\underline{\mathbf{0 . 0 0 2}}$ \\
All-cause mortality & $9(17 \%)$ & $12(12.9 \%)$ & 0.660 \\
\hline
\end{tabular}
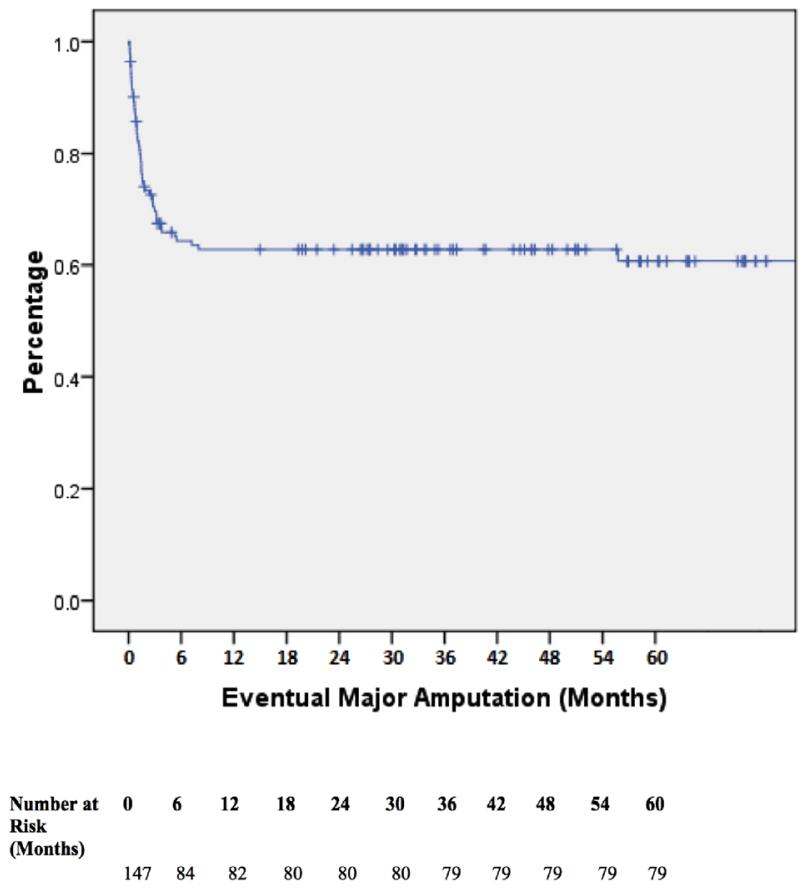

Fig. 1 Overall Limb Salvage. Kaplan-Meier survival curve indicating limb loss for all patients included. Major amputation rates at 6-months, 12-months, 24-months, 36-months, 48-months, and 60-months were $35.4 \%, 36.7 \%, 36.7 \%$, $37.4 \%, 37.4 \%$, and $37.4 \%$ respectively.

patients had an open bypass procedure, while the rest of the revascularisations were performed via percutaneous angioplasty. At post-revascularisation, all patients had an in-line flow to the foot, with at least a single-vessel belowknee run-off. Anterior tibial artery (ATA), posterior tibial artery (PTA), peroneal artery, and plantar arch patency were $44 \%, 40 \%, 53 \%$, and $30 \%$, respectively. Patients with below-knee 3 -vessels, 2 -vessels and 1-vessel run-off were $16 \%, 29 \%$, and $57 \%$, respectively.

In terms of TMA outcomes, 93 patients $(63 \%)$ had a complete healing of TMA. 35 patients $(24 \%)$ required further surgical debridement while 11 patients $(8 \%)$ required interval split skin grafting after a period of NPWT (Table 2). The mean time for patients to receive a skin graft was 62 days. The need for surgical debridement was not predictive of TMA failure $(\mathrm{p}=0.096)$. However, $54 \mathrm{pa}-$ tients $(37 \%)$ had poor TMA wound healing, which even-
Table 4 Causes of death $(n=21)$

\begin{tabular}{ll}
\hline Urosepsis & $1(4.8 \%)$ \\
Lower limb gangrene & $4(19 \%)$ \\
Pneumonia & $4(19 \%)$ \\
End-stage renal failure & $2(9.5 \%)$ \\
Bleeding gastrointestinal tract & $2(9.5 \%)$ \\
Cerebrovascular accident & $2(9.5 \%)$ \\
Acute myocardial infarction & $4(19 \%)$ \\
\hline
\end{tabular}

tually required a BKA. Multivariate analysis showed that diabetes was the only independent predictor of TMA failure (OR 7.11, $\mathrm{p}=0.064)$. Patients with TMA failure were at an increased risk of developing nosocomial infections $(\mathrm{p}=0.025)$ and have a higher re-admission rate within 30 days $(\mathrm{p}=0.002)($ Table 3$)$. Eventual major amputation rates at 6 -months, 12 -months, 24-months, 36-months, 48 -months and 60 -months were $35.4 \%, 36.7 \%, 36.7 \%$, $37.4 \%, 37.4 \%$, and $37.4 \%$, respectively (Fig. 1 ). All-cause mortality rates at 12 -months, 24-months, 36-months, 48 -months, and 60 -months were $12.2 \%, 14.3 \%, 14.3 \%$, $14.3 \%$, and $14.3 \%$, respectively. Only four patients died from sepsis resulting from lower limb gangrene, while other patients passed away from the complications relating to diabetes and chronic diseases (Table 4).

\section{Discussion}

McKittrick was the first researcher to describe TMA in 1949 who reported it as a limb salvage procedure for diabetic patients. ${ }^{12)}$ It was traditionally described as an amputation procedure, including the metatarsal plantar mobilized for flap coverage. Arterial reconstruction and careful selection of patients showed decent healing rates of up to $46 \%$ after 5 years. ${ }^{13)}$ However, there are still a large number of patients who suffered from skin breakdowns after TMA, ${ }^{14)}$ leading to over closing of a skin flap on the wound. In this wise, NPWT has been shown to significantly improve wound healing rates. ${ }^{15)}$ Our institution preference is to leave the wound open after amputation and initiate NPWT. Most patients had significant gangrene, limiting the availability of healthy skin for flap closure. The primary closure also results in significant wound tension. In addition, most of the patients were diabetic and 
at higher risks of wound infections. ${ }^{16)}$ Our institution has since adopted this procedure, preferring to use NPWT to encourage granulation before considering skin grafting for selected patients.

More proximal amputations, such as a BKA or aboveknee amputation (AKA), usually have a higher healing rate. ${ }^{9)}$ However, the drawback is the higher energy expenditure required for ambulating with a subsequent prosthesis. BKA is associated with an acceptable failure rate of $9 \%$ and a risk of failure in patients with end-stage renal failure (ESRF) or previously non-ambulant. ${ }^{17)}$ It is also important to note that these patients have a higher mortality rate, with almost two-thirds of amputees having a contralateral amputation afterward. ${ }^{18)}$

The success TMA's rates reported in the literature are from $27 \%$ to $63 \%{ }^{9,10,19-22)}$ It is important to identify factors predisposing to TMA's failure to reduce the rate of re-admissions that add to both healthcare burden and patient morbidity. Several studies have suggested variable risk factors associated with failure of amputations, which include sepsis, emergency surgery, ESRF, trainee participation, high body mass index (BMI), and long operating duration. ${ }^{10,19,23)}$ However, these are not consistent among various studies, which have been mostly performed in western populations. Thus, one of the objectives of our study was to evaluate TMAs in an Asian population.

Identifying the risk factors for the failure of TMAs is a key interest in addressing the significant re-operation rates. However, the risk factors identified by previous studies are conflicting. ${ }^{20)}$ While critical limb ischemia is clearly a risk factor, ${ }^{9)}$ attempts at using $\mathrm{ABI}$ or angiography results to predict TMA's failure have not been accurate. ${ }^{24)}$ End-stage renal failure is frequently associated with TMA's failure in literature, ${ }^{21)}$ however, it was not found to be significant in this study. Poor glycemic control in diabetic patients has also been shown to be associated with poorer outcomes after amputations, hence prompting some authors to advocate avoiding elective amputations in patients with a HbA1c of over $8 \% .^{25)}$ Although diabetic control was not identified as a significant risk factor in this study, the presence of type II DM was clearly associated with a significantly higher risk of TMA failure.

The incidence of diabetes in Asia is on the rise and it is estimated that more than $60 \%$ of world diabetic patients are in Asia. ${ }^{26)}$ Moreover, the prevalence of diabetes is approximately $11.3 \%$ and expected to increase. ${ }^{11)}$ However, we noted a considerably different prevalence risk factors are identified in this study population versus the average western population with peripheral arterial disease. Almost all our patients had diabetes, and only one-quarter of them were smokers. These differences are important, given that diabetes itself has been shown to contribute to poorer outcomes in patients with PAD. ${ }^{8)}$
Ensuring adequate vascularity is a key important factor for TMAs. Typically, TMA healing rates were shown to improve from $62 \%$ to $83 \%$ after surgical bypass. ${ }^{27)}$ Preoperative and intra-operative assessments are vital in deciding if a TMA is suitable. A retrospective analysis of TMA revealed a $92 \%$ healing rate if patients had all of these 3 factors: minimum ABI of 0.45 in non-diabetics and 0.5 in diabetics, blood total lymphocyte count of $>1.5 \times 10^{3} / \mu \mathrm{L}$ and serum albumin $\geq 3 \mathrm{~g} / \mathrm{dL}$. ${ }^{28)}$ Other studies suggest other modalities for wound healing, such as transcutaneous oxygen pressure $\left(\mathrm{TcPO}_{2}\right),{ }^{29)}$ where revascularisation is recommended if the $\mathrm{TcPO}_{2}$ is $<30 \mathrm{mmHg}$. ${ }^{30)}$

In the current study, all patients received at least one vascular imaging modality (mostly arterial duplex ultrasounds) before amputation. This enables clinicians to offer revascularisation pre-operatively to enhance wound healing rates. Most of the patients underwent percutaneous angioplasty performed by both vascular surgeons and Interventional radiologists at this institution. Analyses of the vascular patency of the affected limbs revealed a trend toward a higher failure rate in patients with the more significant infra-popliteal disease, but did not reach statistical significance. We also analyzed the number of occluded infra-popliteal arteries, but this also did not reveal any significant difference. The pedal arch patency is known to affect wound healing rates, ${ }^{31)}$ but this was again not significantly different between both groups. Toe pressures were also analyzed in both groups, with a cutoff of $<50 \mathrm{mmHg}$, which was generally indicative of poorer outcomes. This is in accordance with the International Working Group for the Diabetic Foot (IWGDF), guidelines for the management of diabetic foot ulcers. ${ }^{32)}$ However, this also did not show any statistical difference.

The risks and benefits of TMA in diabetic limb salvage should be individualized for each patient. TMA should still be considered as it results in ultimately good function and has a reasonable success rate of $63 \%$ according to our results. However, the patient should be well counseled on the risks of failure, and revascularisation should be performed to improve vascularity. TMA should also only be offered to patients who will enjoy functional benefits from limb salvage.

This study has several limitations. Given its retrospective nature, we were unable to determine how patients were selected for TMA. This is important for the decision for TMA encompasses many different factors, including clinical, biochemical, and social factors. Although data were collected over a 7-year period, our sample size is still relatively small. This could lead to insufficient statistical power to identify important associations. Despite these limitations, this study still serves as an important TMA's review in an Asian context. Further studies will be required to identify the risk factors to improve our selection 
of patients undergoing TMA.

\section{Conclusion}

The success rate for PAD limb salvage TMA was 63\% and the presence of diabetes is an independent predictor of TMA failure. As patients with TMA failure were at increased risks of nosocomial infections and 30-day re-admissions, the risks and benefits of TMA for diabetic foot limb salvage must be individualized for each patient.

\section{Disclosure Statement}

All authors have no conflict of interest. This research did not receive any specific grant from funding agencies in the public, commercial, or not-for-profit sectors.

\section{Author Contributions}

Study conception: ZJL, MNAT

Data collection: MNAT, SHL, RMT

Analysis: MNAT, SHL, ZJL

Investigation: MNAT, SHL, ZJL

Writing: MNAT, SHL, ZJL

Funding acquisition: nil

Critical review and revision: all authors

Final approval of the article: all authors

Accountability for all aspects of the work: all authors

\section{References}

1) Kullo IJ, Rooke TW. Peripheral artery disease. N Engl J Med 2016; 374: 861-71.

2) Vos T, Flaxman AD, Naghavi M, et al. Years lived with disability (YLDs) for 1160 sequelae of 289 diseases and injuries 1990-2010: a systematic analysis for the Global Burden of Disease Study 2010. Lancet 2012; 380: 2163-96.

3) An epidemic of risk factors for cardiovascular disease. Lancet 2011; 377: 527.

4) Smith GD, Shipley MJ, Rose G. Intermittent claudication, heart disease risk factors, and mortality. The Whitehall study. Circulation 1990; 82: 1925-31.

5) Fowkes FGR, Rudan D, Rudan I, et al. Comparison of global estimates of prevalence and risk factors for peripheral artery disease in 2000 and 2010: a systematic review and analysis. Lancet 2013; 382: 1329-40.

6) Dumville JC, Lee AJ, Smith FB, et al. The health-related quality of life of people with peripheral arterial disease in the community: the Edinburgh Artery Study. Br J Gen Pract 2004; 54: 826-31.

7) Rhee SY, Guan H, Liu ZM, et al. Multi-country study on the prevalence and clinical features of peripheral arterial disease in Asian type 2 diabetes patients at high risk of atherosclerosis. Diabetes Res Clin Pract 2007; 76: 82-92.

8) Prompers L, Schaper N, Apelqvist J, et al. Prediction of outcome in individuals with diabetic foot ulcers: focus on the differences between individuals with and without peripheral arterial disease. The EURODIALE Study. Diabetologia 2008; 51: 747-55.

9) Hosch J, Quiroga C, Bosma J, et al. Outcomes of transmetatarsal amputations in patients with diabetes mellitus. J Foot Ankle Surg 1997; 36: 430-4.

10) O’Brien PJ, Cox MW, Shortell CK, et al. Risk factors for early failure of surgical amputations: an analysis of 8,878 isolated lower extremity amputation procedures. J Am Coll Surg 2013; 216: 836-42; discussion, 842-4.

11) Ministry of Health, Singapore. Statistics of Disease Burden in Singapore 2010. Available from: https://www.moh.gov.sg/ content/moh_web/home/statistics/Health_Facts_Singapore/ Disease_Burden.html.

12) McKittrick LS, McKittrick JB, Risley TS. Transmetatarsal amputation for infection or gangrene in patients with diabetes mellitus. Ann Surg 1949; 130: 826-40.

13) Wheelock FC Jr. Transmetatarsal amputations and arterial surgery in diabetic patients. N Engl J Med 1961; 264: 31620.

14) Mueller MJ, Allen BT, Sinacore DR. Incidence of skin breakdown and higher amputation after transmetatarsal amputation: implications for rehabilitation. Arch Phys Med Rehabil 1995; 76: 50-4.

15) Armstrong DG, Lavery LA. Negative pressure wound therapy after partial diabetic foot amputation: a multicentre, randomised controlled trial. Lancet 2005; 366: 1704-10.

16) Miyahara T, Suhara M, Nemoto $Y$, et al. Long-term results of treatment for critical limb ischemia. Ann Vasc Dis 2015; 8: 192-7.

17) Wu JT, Wong M, Lo ZJ, et al. A series of 210 peripheral arterial disease below-knee amputations and predictors for subsequent above-knee amputations. Ann Vasc Dis 2017; 10: 217-22.

18) Whitehouse FW, Jurgensen C, Block MA. The later life of the diabetic amputee: another look at fate of the second leg. Diabetes 1968; 17: 520-1.

19) Beaulieu RJ, Grimm JC, Lyu H, et al. Rates and predictors of readmission after minor lower extremity amputations. J Vasc Surg 2015; 62: 101-5.

20) Thorud JC, Jupiter DC, Lorenzana J, et al. Reoperation and reamputation after transmetatarsal amputation: a systematic review and meta-analysis. J Foot Ankle Surg 2016; 55: 1007 12.

21) Landry GJ, Silverman DA, Liem TK, et al. Predictors of healing and functional outcome following transmetatarsal amputations. Arch Surg 2011; 146: 1005-9.

22) Anthony T, Roberts J, Modrall JG, et al. Transmetatarsal amputation: assessment of current selection criteria. Am J Surg 2006; 192: e8-11.

23) Pollard J, Hamilton GA, Rush SM, et al. Mortality and morbidity after transmetatarsal amputation: retrospective review of 101 cases. J Foot Ankle Surg 2006; 45: 91-7.

24) Toursarkissian B, Hagino RT, Khan K, et al. Healing of transmetatarsal amputation in the diabetic patient: is angiography predictive? Ann Vasc Surg 2005; 19: 769-73.

25) Younger AS, Awwad MA, Kalla TP, et al. Risk factors for failure of transmetatarsal amputation in diabetic patients: a cohort study. Foot Ankle Int 2009; 30: 1177-82.

26) Nanditha A, Ma RC, Ramachandran A, et al. Diabetes in 
Asia and the Pacific: implications for the global epidemic. Diabetes Care 2016; 39: 472-85.

27) Miller N, Dardik H, Wolodinger F, et al. Transmetatarsal amputation: the role of adjunctive revascularization. J Vasc Surg 1991; 13: 705-11.

28) Pinzur M, Kaminsky M, Sage R, et al. Amputations at the middle level of the foot. A retrospective and prospective review. J Bone Joint Surg Am 1986; 68: 1061-4.

29) Bunt TJ, Holloway GA. $\mathrm{TcPO}_{2}$ as an accurate predictor of therapy in limb salvage. Ann Vasc Surg 1996; 10: 224-7.
30) Masaki H, Tabuchi A, Yunoki Y, et al. Collective therapy and therapeutic strategy for critical limb ischemia. Ann Vasc Dis 2013; 6: 27-32.

31) Rashid H, Slim H, Zayed H, et al. The impact of arterial pedal arch quality and angiosome revascularisation on foot tissue loss healing and infrapopliteal bypass outcome. J Vasc Surg 2013; 57: 1219-26.

32) Bakker K, Apelqvist J, Schaper NC. Practical guidelines on the management and prevention of the diabetic foot 2011. Diabetes Metab Res Rev 2012; 28 Suppl 1: 225-31. 